\title{
Association of TM6SF2 rs58542926 gene polymorphism with the risk of non- alcoholic fatty liver disease and colorectal adenoma in Chinese Han population
}

Yuan $\mathrm{Li}^{1,2}$, Shousheng Liu, ${ }^{4,5}$, Yuqiang Gao ${ }^{2}$, Huan $\mathrm{Ma}^{2}$, Shuhui Zhan ${ }^{2}$, Yan Yang ${ }^{2}$, Yongning Xin ${ }^{1,2,3,5^{*}}$ (i) and Shiying Xuan ${ }^{1,2,5^{*}}$

\begin{abstract}
Background: Genetic factors affect the risk of non-alcoholic fatty liver disease (NAFLD) and colorectal adenoma (CRA) importantly. Transmembrane protein 6 superfamily member 2 (TM6SF2) rs58542926 is a significant genetic susceptibility site for NAFLD. The relationships of TM6SF2 rs58542926 with the risk of NAFLD and CRA in Chinese Han population were unclear. The aim of this study was to investigate the association of TM6SF2 rs58542926 with the risk of NAFLD and CRA, and the effect of CRA on TM6SF2 rs58542926 carried NAFLD patients.

Results: A total of 839 Chinese Han population were included in this retrospective study. TM6SF2 rs58542926 polymorphism was genotyped in B-type ultrasonography proven NAFLD patients with or without CRA, CRA patients and healthy controls, using polymerase chain reaction. Serum lipid profiles were determined using biochemical methods. Statistical analyses were performed using SPSS statistical software, version 16.0 for mac. There was a significant difference in the distribution of genotype and allele of TM6SF2 rs58542926 in NAFLD and NAFLD\&CRA patients compared to controls. The CT + TT genotypes were tightly associated with the risk of NAFLD and NAFLD\&CRA. TM6SF2 rs58542926 T allele promotes the abnormal regulation of lipids metabolism and liver injury in NAFLD patients and NAFLD\&CRA patients. CRA aggravates the clinical performance of NAFLD in T allele carriers.

Conclusions: We demonstrated the significant association between TM6SF2 rs58542926 polymorphism and the risk of NAFLD and NAFLD\&CRA in a Chinese Han population. The TM6SF2 rs58542926 T allele promotes the abnormal regulation of lipid profiles and liver injury in NAFLD patients, NAFLD\&CRA patients, and overall subjects.
\end{abstract}

Keywords: TM6SF2, rs58542926, NAFLD, Colorectal adenomas

\section{Background}

NAFLD is one of the most common chronic liver diseases in the world [1]. NAFLD is the manifestation of metabolic syndrome in liver, which comprises of simple non-alcoholic fatty liver (NAFL), non-alcoholic steatohepatitis (NASH), fibrosis, and cirrhosis, even the hepatocellular carcinoma (HCC) [2]. In Europe, the prevalence of NAFLD increases year by year due to the risk factors such as obesity and aging [3]. An eight-year follow-up study in China showed

\footnotetext{
* Correspondence: xinyongning@163.com; xuansydxy@163.com ${ }^{1}$ Medical College of Qingdao University, Qingdao 266071, China Full list of author information is available at the end of the article
}

that NAFLD was prevalent in common population and the increased tendency of NAFLD was obviously [4].

In recent years, genome wide association studies (GWAS) have been conducted to explore the significant SNP (single nucleotide polymorphism) sites in genome which were associated NAFLD and lipid metabolism. Several significant genetic susceptibility genes such as PNPLA3, KLF6, GCKR, LYPLAL1, had been proven the key roles in the development of NAFLD in different regions and ethnicities [5-10]. TM6SF2 rs58542926 is a substitution of guanine by adenine in nucleotide 499, that leads to the replacement of glutamic acid by lysine in amino acid residue 167 (E167K). In 2014, the significance of TM6SF2 rs58542926 polymorphism in

(c) The Author(s). 2019 Open Access This article is distributed under the terms of the Creative Commons Attribution 4.0 International License (http://creativecommons.org/licenses/by/4.0/), which permits unrestricted use, distribution, and reproduction in any medium, provided you give appropriate credit to the original author(s) and the source, provide a link to the Creative Commons license, and indicate if changes were made. The Creative Commons Public Domain Dedication waiver (http://creativecommons.org/publicdomain/zero/1.0/) applies to the data made available in this article, unless otherwise stated. 
NAFLD was found for the first time [11]. Subsequent studies had proven that TM6SF2 rs58542926 was a significant risk factor for the development of NAFLD. Chen et al. systematic reviewed the genetic susceptibility of TM6SF2 rs58542926 in NAFLD [12]. Giovanni et al. proved that TM6SF2 rs58542926 could affect nutrient oxidation, glucose homeostasis, and postprandial lipoprotein, adipokine in NAFLD patients [13]. In addition, the effect of TM6SF2 rs58542926 on serum lipid levels in obese children was investigated by Grandone et al., the results showed that TM6SF2 rs58542926 was significant associated with the lower level of low density lipoprotein cholesterol, and could promote liver injury in obese children [14]. In China, the key effect of TM6SF2 rs58542926 on NAFLD in Shanghai district and Hong Kong was reported by Wong and Wang, that provided a primary evidence for the key role of TM6SF2 rs58542926 in Asian especially in Chinese $[15,16]$.

Colorectal cancer (CRC) is one of the most prevalent malignancies in the world, the incidence of CRC has risen steadily in recent years $[17,18]$. CRA is a benign precursor of CRC, polyps - adenoma - cancer sequence has been recognized as the typical patterns of CRC $[19,20]$. Accumulated evidences have shown that NAFLD and metabolic syndrome were tightly associated with CRA, and abnormal lipid metabolism was the significant characteristic in the patients with NAFLD and CRA [21-24]. Some polymorphism sites such as $A D H 1 B$ Arg47His, NAT1 rs65867, and the gene polymorphisms in WNT6 and WNT10A in patients with CRA had been studied, the results suggested that the risk of CRA was markedly associated with gene genetic susceptibility [25-27]. In view of the tight association of CRA and NAFLD, the role of TM6SF2 rs58542926 in the risk of CRA was unknown. The purpose of this study was to investigate the relationship of TM6SF2 rs58542926 with the risk of NAFLD and CRA in Qingdao district of China and explore the effect of CRA on the TM6SF2 rs58542926 carried NAFLD patients.

\section{Methods}

\section{Subjects}

This study was approved by the ethics committee of Qingdao municipal hospital and strictly in accordance with the Helsinki declaration and its appendices [28]. Each patient was signed the written informed consent before participating in this study. All the subjects were recruited in the Health examination center and department of gastroenterology of Qingdao municipal hospital from March 2016 to March 2018, and all the subjects are the unrelated Chinese Han population. The essential information such as name, gender, age, was obtained using a standard questionnaire.
All patients were subjected to the colonoscopy by three experienced endoscopists with at least 5 years' experience in colonoscopy examination at the Qingdao municipal hospital. Before colonoscopy examination, subjects took the polyethylene glycol electrolyte powder orally to prepare the bowel. The quality of bowel preparation was evaluated according to the intestinal residual contents and surface seen as good (more than 95\% surface seen and without or small volume of clear liquid), poor (less than $90 \%$ surface seen, and semi-solid stool presented that could not be suctioned or washed away), and fair (between good and poor). Endoscope (OLYMPUS 260, Japan) reached caecum was defined as a complete colonoscopy examination. Subjects with an incomplete colonoscopy examination would be excluded from this study. The CRA were defined as a benign tumor originating from the glandular epithelium of colorectal mucosa, including colon adenoma and rectal adenoma. Subjects as below were excluded: 1) suffering from tumors or surgery in nearly 2 years; 2) suffering from inflammatory bowel disease or chronic liver disease or kidney disease; 3) possessing a history of heart failure; 4) with autoimmune disorders (rheumatoid arthritis, AIDS); 5) long-term use of immunosuppression preparations; 6) suffering from asthma, COPD or other pulmonary diseases; 7) familial polyposis.

The diagnosis of NAFLD was conducted according to the criteria of American association for the study of liver diseases (AASLD) [29]. All the NAFLD patients were diagnosed 10 times repeatedly at the same point by two experienced physicians. FibroScan $C A P$ value was obtained automatically by taking the median. Patients with other causes such as high alcohol intake (males $>210 \mathrm{~g} / \mathrm{w}$, females $>140 \mathrm{~g} / \mathrm{w}$ ), autoimmune hepatitis, viral hepatitis, drug-induced hepatitis, various liver cirrhosis and alcoholic liver disease were also excluded. Healthy controls were confirmed by the same examinations at Qingdao municipal hospital. According to the diagnostic results, subjects were divided into four groups: NAFLD group, NAFLF \& CRA group, CRA group, and healthy control group.

\section{Biochemical analyses}

Blood sample was taken from the median vein of each subject with a 12-h overnight fasting and placed into the ethylene diamine tetraacetic acid (EDTA)-containing tube. The body mass index (BMI) of each subject was calculated equals to mass $(\mathrm{kg}) /$ height $(\mathrm{m})^{2}$. For biochemical analyses, serum levels of fasting blood glucose (FBG), total cholesterol (TC), triglyceride (TG), low density lipoprotein cholesterol (LDL-C), high density lipoprotein cholesterol (HDL-C), aspartate aminotransferase (AST), alanine transaminase (ALT), uric acid (UA), were measured by standard clinical laboratory techniques (IChem-540 automatic biochemical analyzer, Shenzhen, China), respectively. 


\section{Genomic DNA extraction and genotyping}

Genomic DNA was extracted from blood sample using the Genomic DNA purification Kit (Beijing Bioteke Biotechnology, Beijing, China) as described by [30]. Polymerase chain reaction (PCR) was performed to test the genotype of TM6SF2 rs58542926 of all the subjects using the primers: 5'-ACGTTGGATGTGAAGACCTTCATGCCA GCC-3' and 5'-ACGTTGGATGGCACCATGGAAGGCA AATAC-3'. PCR amplification (Labnet, United States) was performed as the following program: $95^{\circ} \mathrm{C}$ for $10 \mathrm{~min}$, followed by 35 cycles: denaturation at $94^{\circ} \mathrm{C}$ for $3 \mathrm{~min}$, annealing at $58{ }^{\circ} \mathrm{C}$ for $1 \mathrm{~min}$ and extension at $72{ }^{\circ} \mathrm{C}$ for 1 min. All PCR products were resolved using $2 \%$ agarose gel electrophoresis at $120 \mathrm{~V}$ for $30 \mathrm{~min}$ and stained with ethidium bromide. DNA sequencing was performed to identify the genotype of TM6SF2 rs58542926 using the ABI Prism sequence detection system ABI3730 (Foster city, CA, USA), and the SNP genotyping success rates were $>95 \%$. All Genotyping were performed in blinded fashion.

\section{Data analysis}

Statistical analyses were performed using SPSS (version 16.0 for Mac) statistical software as previously described [30]. The Hardy-Weinberg equilibrium between expected and observed genotype distribution and the distributions of genotype between patients and controls were analyzed by Pearson's $X^{2}$ test.

Genotype and allele frequencies were investigated by counting the DNA sequencing data of each subject. Clinical and biochemical characteristics of each patient were shown as mean \pm standard deviation (SD) and the differences of characteristics in different groups were tested using the $\chi^{2}$ test, student's $t$-test or paired samples $t$-test. The association between polymorphism and presence/absence of NAFLD/CRA was evaluated by logistic regression analysis and estimated by the odds ratio (OR) with $95 \%$ confidence interval $(\mathrm{CI}) . P<0.05$ was considered as statistically significant.

\section{Results}

\section{Characteristics of the study population}

A total of 839 subjects were included in this study, which including 201 patients (128 males and 73 females) with NAFLD, 188 patients (127 males and 61 females) with NAFLD\&CRA, 211 patients (134 males and $77 \mathrm{fe-}$ males) with CRA, and 239 healthy control subjects (133 males and 106 females). The clinical characteristics of all the subjects were shown in the Table 1 . As expected, the traditional risk factors of NAFLD, such as BMI, CAP, FPG, TG, AST, ALT, and UA were significant higher in the NAFLD group and NAFLD\&CRA group compared to healthy control group (all $P<0.05$ ). In CRA group, the serum levels of BMI, CAP, FPG, TG, and UA were significantly higher than in healthy control group (all $P<0.05)$. Compared to the CRA group, the higher serum levels of CAP and ALT in NAFLD group, and the higher serum levels of CAP and AST in NAFLD\&CRA group were observed (all $P<0.05$ ). No significant differences of all the clinical characteristics were observed between NAFLD group and NAFLD\&CRA group (Table 1).

\section{Distribution of genotype and allele}

The genotype distributions of TM6SF2 rs58542926 were in consistence with the Hardy-Weinberg ( $\mathrm{H}-\mathrm{W})$ equilibrium in all the four groups (all $P>0.05$ ) (Table 2). The

Table 1 Clinical and biochemical characteristics of each group patients ${ }^{a}$

\begin{tabular}{|c|c|c|c|c|}
\hline Characteristic & $\begin{array}{l}\text { NAFLD } \\
(n=201)\end{array}$ & $\begin{array}{l}\text { NAFLD\&CRA } \\
(n=188)\end{array}$ & $\begin{array}{l}\text { CRA } \\
(n=211)\end{array}$ & $\begin{array}{l}\text { Control } \\
(n=239)\end{array}$ \\
\hline Age (yr) & $54.80 \pm 0.56$ & $54.60 \pm 0.48$ & $55.10 \pm 0.52$ & $54.00 \pm 0.78$ \\
\hline $\mathrm{BMI}\left(\mathrm{kg} / \mathrm{m}^{2}\right)$ & $24.56 \pm 1.28^{*}$ & $25.45 \pm 2.07^{*}$ & $24.12 \pm 1.43^{*}$ & $23.05 \pm 0.67$ \\
\hline CAP $(\mathrm{dB} / \mathrm{m})$ & $242.56 \pm 31.42^{* @}$ & $245.64 \pm 29.63^{* @}$ & $230.66 \pm 32.33^{*}$ & $201.23 \pm 28.67$ \\
\hline FPG (mmol/L) & $5.01 \pm 0.45^{*}$ & $5.24 \pm 0.89^{*}$ & $5.09 \pm 0.78^{*}$ & $4.78 \pm 0.99$ \\
\hline $\mathrm{TC}(\mathrm{mmol} / \mathrm{L})$ & $5.49 \pm 0.95$ & $5.56 \pm 0.87$ & $5.46 \pm 0.78$ & $5.43 \pm 0.79$ \\
\hline $\mathrm{TG}(\mathrm{mmol} / \mathrm{L})$ & $1.96 \pm 1.23^{*}$ & $1.98 \pm 1.34^{*}$ & $1.89 \pm 1.27^{*}$ & $1.48 \pm 1.06$ \\
\hline $\mathrm{LDL}-\mathrm{C}(\mathrm{mmol} / \mathrm{L})$ & $3.32 \pm 0.45$ & $3.31 \pm 0.79$ & $3.28 \pm 0.67$ & $3.20 \pm 0.77$ \\
\hline $\mathrm{HDL}-\mathrm{C}(\mathrm{mmol} / \mathrm{L})$ & $1.33 \pm 0.32$ & $1.32 \pm 0.37$ & $1.37 \pm 0.56$ & $1.38 \pm 0.41$ \\
\hline AST (U/L) & $39.60 \pm 8.30^{*}$ & $40.50 \pm 9.2^{* @}$ & $34.50 \pm 8.50$ & $31.40 \pm 7.30$ \\
\hline ALT (U/L) & $37.20 \pm 9.90^{* @}$ & $39.90 \pm 10.30^{*}$ & $31.80 \pm 11.90$ & $30.90 \pm 10.10$ \\
\hline UA ( $\mu \mathrm{mol} / \mathrm{L})$ & $368.76 \pm 80.32^{*}$ & $370.58 \pm 67.92^{*}$ & $369.61 \pm 84.26^{*}$ & $340.56 \pm 75.67$ \\
\hline
\end{tabular}

Abbreviations: NAFLD non-alcoholic fatty liver disease, CRA colorectal adenoma, BMI body mass index, CAP controlled attenuation parameter, FPG fasting plasma glucose, TC total cholesterol, TG triglyceride, LDL-C low-density lipoprotein cholesterol, HDL-C high-density lipoprotein cholesterol, AST aspartate aminotransferase, ALT alanine aminotransferase, $U A$ uric acid

"compared with the control group, $P<0.05 ;{ }^{\circledR}$ compared with CRA group, $P<0.05$

${ }^{\mathrm{a}}$ Data are presented as mean $\pm \mathrm{SD}$ 
Table 2 Results of the Hardy-Weinberg (H-W) Equilibrium ${ }^{a}$

\begin{tabular}{lll}
\hline Group & $x^{2}$ & $P$ value \\
\hline NAFLD & 0.2245 & 0.64 \\
NAFLD\&CRA & 0.2411 & 0.62 \\
CRA & 0.0600 & 0.81 \\
Control & 0.0267 & 0.87 \\
\hline
\end{tabular}

${ }^{\mathrm{a}}$ Data were compared by chi-square test

variant rs58542926T allele frequencies in NAFLD group, NAFLD\&CRA group, CRA group, and healthy control group were $3.2,3.5,1.7$, and $1.0 \%$, respectively (Table 3 ). To ensure the accuracy of our genotyping, 100 samples were randomly selected for reverse sequencing, the success rate of duplicated genotyping was $100 \%$. There were significant differences of rs58542926 genotype distributions in the NAFLD group vs control group, NAFLD\&CRA group vs control group, and NAFLD group vs CRA group (all $P<0.05$ ), no significant differences of rs58542926 genotype distributions were observed in the CRA group vs control group, NAFLD\&CRA group vs NAFLD group, and NAFLD\&CRA vs CRA group. Similar, the allele distributions of rs58542926 in NAFLD group vs control group, NAFLD\&CRA group vs control group were significant different (both $P<0.05$ ), no significant differences of rs58542926 allele distributions were observed in the CRA group vs control group, NAFLD\&CRA group vs NAFLD group, NAFLD\&CRA vs CRA group, and NAFLD group vs CRA group (Table 3 ). Presence of $\mathrm{CT}+\mathrm{TT}$ genotype markedly associated the risk of NAFLD (OR: 1.464, 95\%CI: 1.300-1.599, $P=0.013$ ), the significant association of $\mathrm{CT}+\mathrm{TT}$ genotype with the risk of NAFLD\&CRA was also observed in NAFLD\&CRA group (OR: 1.235, 95\%CI: 1.083-1.801, $P=0.027$ ).
Presence of CT + TT genotype did not associate with the risk of CRA (OR: 1.053, 95\% CI: 0.986-1.599, $P=0.231$ ). After adjusting for gender, age, and BMI, the association of TM6SF2 rs58542926 CT + TT genotype with the risk of NAFLD and NAFLD\&CRA were still significant (OR: 1.368, 95\% CI: 1.113-1.504, $P=0.025$; OR: 1.129, 95\% CI: $1.002-1.768, P=0.038$, respectively) (Table 4 ).

\section{Association of TM6SF2 rs58542926 T allele with the clinical parameters in each group and overall subjects} To investigate whether the TM6SF2 rs58542926 T allele are correlated with clinical parameters, we compared the clinical parameters in $\mathrm{T}$ allele carriers and non-carriers in each group and overall subjects. As the results shown in the Table 5, in NAFLD group, $\mathrm{T}$ allele carriers had the higher levels of CAP and ALT, and lower level of TG than non-carriers (all $P<0.05$ ). In NAFLD\&CRA group, the higher levels of CAP, AST, and ALT, and lower levels of TC, TG, and LDL-C were observed in T allele carriers when compared to non-carriers (all $P<0.05$ ). However, no significant differences of all the clinical parameters were observed between the $\mathrm{T}$ allele carriers and non-carriers in the CRA group and healthy control group (all $P>0.05$ ). In overall subjects, $\mathrm{T}$ allele carriers possessed the higher CAP value and serum AST level, and the lower serum TG level than non-carriers (Table 6).

\section{Discussion}

NAFLD as the most prevalent chronic liver disease is affected by many risk factors such as obesity, insulin resistance, oxidative stress, and genetic factors [31, 32]. Increased understanding of the underlying mechanism in genetics for the development of NAFLD was implemented [33, 34]. TM6SF2 is a novel genetic susceptibility

Table 3 Distribution of genotypes and allele frequencies of TM6SF2 rs58542926 in each group ${ }^{a, b}$

\begin{tabular}{|c|c|c|c|c|c|c|c|c|c|}
\hline \multirow[t]{2}{*}{ Group } & \multicolumn{5}{|l|}{ Genotype } & \multicolumn{4}{|l|}{ Allele } \\
\hline & $\overline{C C}$ & $C T$ & $\pi$ & $x^{2}$ & $P$ value & C & $\mathrm{T}$ & $x^{2}$ & $P$ value \\
\hline NAFLD & $188(93.5 \%)$ & $13(6.5 \%)$ & $0(0.0 \%)$ & 5.328 & 0.021 & $389(96.8 \%)$ & $13(3.2 \%)$ & 5.238 & 0.022 \\
\hline Control & 234 (97.9\%) & $5(2.1 \%)$ & $0(0.0 \%)$ & & & 474 (98.9\%) & $5(1.1 \%)$ & & \\
\hline NAFLD\&CRA & 175 (93.1\%) & $13(6.9 \%)$ & $0(0.0 \%)$ & 6.062 & 0.014 & $363(96.5 \%)$ & 13 (3.5\%) & 5.954 & 0.015 \\
\hline Control & 234 (97.9\%) & $5(2.1 \%)$ & $0(0.0 \%)$ & & & 474 (98.9\%) & $5(1.1 \%)$ & & \\
\hline CRA & 204 (96.7\%) & $7(3.3 \%)$ & $0(0.0 \%)$ & 0.648 & 0.421 & 415 (98.3\%) & $7(1.7 \%)$ & 0.646 & 0.422 \\
\hline Control & 234 (97.9\%) & $5(2.1 \%)$ & $0(0.0 \%)$ & & & 474 (98.9\%) & $5(1.1 \%)$ & & \\
\hline NAFLD\&CRA & 175 (93.1\%) & $13(6.9 \%)$ & $0(0.0 \%)$ & 0.031 & 0.860 & $363(96.5 \%)$ & $13(3.5 \%)$ & 0.030 & 0.862 \\
\hline NAFLD & 188 (93.5\%) & $13(6.5 \%)$ & $0(0.0 \%)$ & & & 389 (96.8\%) & $13(3.2 \%)$ & & \\
\hline NAFLD\&CRA & 175 (93.1\%) & $13(6.9 \%)$ & $0(0.0 \%)$ & 2.702 & 0.100 & $363(96.5 \%)$ & $13(3.5 \%)$ & 2.633 & 0.105 \\
\hline CRA & 204 (96.7\%) & $7(3.3 \%)$ & $0(0.0 \%)$ & & & 415 (98.3\%) & $7(1.7 \%)$ & & \\
\hline NAFLD & 188 (93.5\%) & $13(6.5 \%)$ & $0(0.0 \%)$ & 4.054 & 0.044 & 389 (96.8\%) & $13(3.2 \%)$ & 2.439 & 0.118 \\
\hline CRA & 204 (96.7\%) & 7 (3.3\%) & $0(0.0 \%)$ & & & 415 (98.3\%) & $7(1.7 \%)$ & & \\
\hline
\end{tabular}

${ }^{a}$ Data were compared by chi-square test

${ }^{b}$ Values are expressed as No. (\%) 
Table 4 Association of genotypes with NAFLD, NAFLD\&CRA and CRA groups ${ }^{a}$

\begin{tabular}{|c|c|c|c|c|c|c|}
\hline \multirow[t]{2}{*}{ Group } & \multicolumn{3}{|c|}{ Unadjusted } & \multicolumn{3}{|l|}{ Adjusted } \\
\hline & Genotype & OR $(95 \% C l)$ & $P$ value & Genotype & OR $(95 \%$ Cl) & $P$ value \\
\hline \multirow[t]{2}{*}{ NAFLD } & CC & 1 & 0.013 & CC & 1 & 0.025 \\
\hline & $C T+\pi$ & $1.464(1.300-1.599)$ & & $C T+\pi$ & $1.368(1.113-1.504)$ & \\
\hline \multirow[t]{2}{*}{ NAFLD\&CRA } & $\mathrm{CC}$ & 1 & 0.027 & $\mathrm{CC}$ & 1 & 0.038 \\
\hline & $\mathrm{CT}+\mathrm{T}$ & $1.235(1.083-1.801)$ & & $\mathrm{CT}+\mathrm{TT}$ & $1.129(1.002-1.768)$ & \\
\hline \multirow[t]{2}{*}{ CRA } & $\mathrm{CC}$ & 1 & 0.231 & $\mathrm{CC}$ & 1 & 0.202 \\
\hline & $\mathrm{CT}+\mathrm{TT}$ & $1.053(0.986-1.599)$ & & $\mathrm{CT}+\mathrm{TT}$ & $1.209(0.954-1.783)$ & \\
\hline
\end{tabular}

The multiple-logistic regression model was adjusted for gender, age, and BMI

gene and accumulated attentions have been paid to the association of TM6SF2 rs58542926 with the risk of NAFLD [12, 35], but the studies conducted in Chinese Han population were remain insufficient. Some reports showed that CRA was tightly associated with the risk of
NAFLD, and the incidence of NAFLD combined with CRA was increasing in recent years [36, 37]. In this study, we investigated the association of TM6SF2 rs58542926 polymorphism with the risk of NAFLD in Han population of Qingdao district in China, and explored the effect of

Table 5 Clinical characteristics of TM6SF2 rs58542926 T carriers and non-carriers in each group ${ }^{a}$

\begin{tabular}{|c|c|c|c|c|c|c|}
\hline & \multicolumn{3}{|l|}{ NAFLD } & \multicolumn{3}{|l|}{ NAFLD\&CRA } \\
\hline & $\begin{array}{l}\text { Carriers } \\
(n=13)\end{array}$ & $\begin{array}{l}\text { Non-carriers } \\
(n=188)\end{array}$ & $P$ value & $\begin{array}{l}\text { Carriers } \\
(n=13)\end{array}$ & $\begin{array}{l}\text { Non-carriers } \\
(n=175)\end{array}$ & $P$ value \\
\hline Age (yr) & $53.80 \pm 0.46$ & $55.00 \pm 0.51$ & 0.534 & $54.90 \pm 0.61$ & $54.30 \pm 0.49$ & 0.411 \\
\hline $\mathrm{BMI}\left(\mathrm{kg} / \mathrm{m}^{2}\right)$ & $24.99 \pm 1.36$ & $23.95 \pm 1.23$ & 0.957 & $24.98 \pm 1.98$ & $26.12 \pm 2.02$ & 0.053 \\
\hline CAP $(\mathrm{dB} / \mathrm{m})$ & $262.34 \pm 32.65$ & $239.11 \pm 37.76$ & 0.031 & $260.21 \pm 22.52$ & $240.46 \pm 21.34$ & 0.001 \\
\hline FPG (mmol/L) & $4.89 \pm 0.98$ & $5.13 \pm 0.12$ & 0.163 & $5.21 \pm 0.32$ & $5.26 \pm 0.79$ & 0.341 \\
\hline $\mathrm{TC}(\mathrm{mmol} / \mathrm{L})$ & $5.01 \pm 0.67$ & $5.63 \pm 0.83$ & 0.352 & $4.80 \pm 0.98$ & $5.61 \pm 0.78$ & 0.013 \\
\hline TG (mmol/L) & $1.49 \pm 1.21$ & $2.09 \pm 0.95$ & 0.031 & $1.50 \pm 1.39$ & $2.12 \pm 1.09$ & 0.001 \\
\hline LDL-C (mmol/L) & $3.38 \pm 0.37$ & $3.29 \pm 0.41$ & 0.571 & $3.12 \pm 0.62$ & $3.36 \pm 0.70$ & 0.039 \\
\hline $\mathrm{HDL}-\mathrm{C}(\mathrm{mmol} / \mathrm{L})$ & $1.39 \pm 0.43$ & $1.24 \pm 0.12$ & 0.368 & $1.30 \pm 0.32$ & $1.35 \pm 0.37$ & 0.276 \\
\hline AST (U/L) & $44.40 \pm 7.90$ & $37.30 \pm 7.60$ & 0.067 & $46.30 \pm 8.90$ & $38.70 \pm 8.70$ & 0.048 \\
\hline $\operatorname{ALT}(\mathrm{U} / \mathrm{L})$ & $43.70 \pm 8.90$ & $35.76 \pm 9.10$ & 0.039 & $45.80 \pm 10.40$ & $37.80 \pm 9.40$ & 0.043 \\
\hline \multirow[t]{3}{*}{$\mathrm{UA}(\mu \mathrm{mol} / \mathrm{L})$} & $363.34 \pm 81.36$ & $373.43 \pm 78.02$ & 0.632 & $372.51 \pm 70.91$ & $367.48 \pm 61.32$ & 0.652 \\
\hline & \multicolumn{3}{|l|}{ CRA } & \multicolumn{3}{|l|}{ Control } \\
\hline & $\begin{array}{l}\text { Carriers } \\
(n=7)\end{array}$ & $\begin{array}{l}\text { Non-carriers } \\
(n=204)\end{array}$ & $P$ value & $\begin{array}{l}\text { Carriers } \\
(n=5)\end{array}$ & $\begin{array}{l}\text { Non-carriers } \\
(n=234)\end{array}$ & $P$ value \\
\hline Age (yr) & $55.90 \pm 0.54$ & $54.90 \pm 0.48$ & 0.853 & $53.80 \pm 0.57$ & $54.20 \pm 0.65$ & 0.967 \\
\hline $\mathrm{BMI}\left(\mathrm{kg} / \mathrm{m}^{2}\right)$ & $24.38 \pm 1.38$ & $23.85 \pm 1.56$ & 0.574 & $22.98 \pm 0.91$ & $23.13 \pm 0.77$ & 0.968 \\
\hline CAP $(\mathrm{dB} / \mathrm{m})$ & $240.66 \pm 34.73$ & $227.66 \pm 30.67$ & 0.195 & $210.45 \pm 30.67$ & $199.48 \pm 25.76$ & 0.698 \\
\hline FPG (mmol/L) & $5.04 \pm 0.45$ & $4.79 \pm 0.71$ & 0.837 & $4.99 \pm 1.03$ & $4.69 \pm 0.68$ & 0.753 \\
\hline $\mathrm{TC}(\mathrm{mmol} / \mathrm{L})$ & $5.29 \pm 0.98$ & $5.54 \pm 0.63$ & 0.111 & $5.27 \pm 0.76$ & $5.74 \pm 0.64$ & 0.386 \\
\hline TG $(\mathrm{mmol} / \mathrm{L})$ & $1.76 \pm 1.28$ & $1.92 \pm 1.37$ & 0.093 & $1.36 \pm 0.98$ & $1.57 \pm 1.21$ & 0.215 \\
\hline LDL-C (mmol/L) & $3.23 \pm 0.75$ & $3.30 \pm 0.63$ & 0.265 & $2.92 \pm 0.97$ & $3.31 \pm 0.67$ & 0.470 \\
\hline $\mathrm{HDL}-\mathrm{C}(\mathrm{mmol} / \mathrm{L})$ & $1.47 \pm 0.63$ & $1.33 \pm 0.59$ & 0.097 & $1.57 \pm 0.31$ & $1.31 \pm 0.54$ & 0.865 \\
\hline AST (U/L) & $36.90 \pm 10.80$ & $33.50 \pm 7.50$ & 0.683 & $30.60 \pm 6.30$ & $32.70 \pm 7.70$ & 0.461 \\
\hline $\operatorname{ALT}(U / L)$ & $30.60 \pm 9.90$ & $32.80 \pm 13.10$ & 0.473 & $26.90 \pm 8.10$ & $32.90 \pm 10.30$ & 0.643 \\
\hline UA $(\mu \mathrm{mol} / \mathrm{L})$ & $358.61 \pm 81.53$ & $379.61 \pm 84.98$ & 0.352 & $335.36 \pm 67.69$ & $351.55 \pm 73.64$ & 0.198 \\
\hline
\end{tabular}

Abbreviations: NAFLD non-alcoholic fatty liver disease, CRA colorectal adenoma, BMI body mass index, CAP controlled attenuation parameter, FPG fasting plasma glucose, TC total cholesterol, TG triglyceride, $L D L-C$ low-density lipoprotein cholesterol, $H D L$ - $C$ high-density lipoprotein cholesterol, $A S T$ aspartate aminotransferase, ALT alanine aminotransferase, $U A$ uric acid

avalues are expressed as mean \pm SD and compared by Student's $t$-test 
Table 6 Clinical characteristics of TM6SF2 rs58542926 T carriers and non-carriers in overall subjects ${ }^{a}$

\begin{tabular}{llll}
\hline Characteristic & $C C(n=794)$ & $C T+T T(n=45)$ & $P$ Value \\
\hline Age $(\mathrm{yr})$ & $54.60 \pm 0.49$ & $54.70 \pm 0.48$ & 0.863 \\
BMI $\left(\mathrm{kg} / \mathrm{m}^{2}\right)$ & $24.26 \pm 1.40$ & $24.33 \pm 1.41$ & 0.841 \\
CAP $(\mathrm{dB} / \mathrm{m})$ & $226.68 \pm 28.88$ & $243.42 \pm 30.14$ & 0.034 \\
FPG $(\mathrm{mmol} / \mathrm{L})$ & $4.97 \pm 0.58$ & $5.03 \pm 0.81$ & 0.741 \\
TC $(\mathrm{mmol} / \mathrm{L})$ & $5.63 \pm 0.72$ & $5.09 \pm 0.85$ & 0.175 \\
TG $(\mathrm{mmol} / \mathrm{L})$ & $1.93 \pm 1.15$ & $1.53 \pm 1.22$ & 0.029 \\
LDL-C $(\mathrm{mmol} / \mathrm{L})$ & $3.32 \pm 0.60$ & $3.16 \pm 0.68$ & 0.492 \\
HDL-C $(\mathrm{mmol} / \mathrm{L})$ & $1.31 \pm 0.41$ & $1.43 \pm 0.42$ & 0.429 \\
AST $(\mathrm{U} / \mathrm{L})$ & $39.66 \pm 8.50$ & $43.93 \pm 7.90$ & 0.042 \\
ALT $(\mathrm{U} / \mathrm{L})$ & $34.82 \pm 10.50$ & $36.57 \pm 9.30$ & 0.093 \\
UA $(\mu \mathrm{mol} / \mathrm{L})$ & $368.03 \pm 74.49$ & $357.46 \pm 75.37$ & 0.735
\end{tabular}

Abbreviations: NAFLD non-alcoholic fatty liver disease, CRA colorectal adenoma, $B M I$ body mass index, CAP controlled attenuation parameter, FPG fasting plasma glucose, $T C$ total cholesterol, $T G$ triglyceride, $L D L-C$ low-density lipoprotein cholesterol, HDL-C high-density lipoprotein cholesterol, AST aspartate aminotransferase, $A L T$ alanine aminotransferase, UA uric acid avalues are expressed as mean \pm SD and compared by Student's $t$-test

CRA on the TM6SF2 rs58542926 carried NAFLD patients. The results in this study showed that patients with NAFLD and NAFLD\&CRA had the higher BMI and CAP values and serum levels of FGP, TG, AST, ALT, and UA than healthy controls. In additional, the higher BMI and CAP values and serum levels of FPG, TG, and UA were observed in patients with CRA compared to healthy controls. In Chinese Han population, TM6SF2 rs58542926 was significant associated with the risk of NAFLD and NAFLD\&CRA, but no with the risk of CRA.

NAFLD is the hepatic manifestation of metabolic syndrome, the abnormal regulation of glucose and lipid metabolism are existed in the NAFLD patients [38]. In recent years, with the development of diagnostic methods for NAFLD, transient elastography (FibroScan ${ }^{\oplus}, \mathrm{TE}$ ) has been used as an accurate non-invasive diagnostic method, the degrees of hepatic steatosis could be reflected by the CAP values $[39,40]$. In our study, the typical performance of metabolic syndrome such as the higher BMI and CAP values, higher serum levels of FPG, TG, AST, ALT, and UA were observed in the NAFLD patients and NAFLD\&CRA patients. Accumulated evidences have shown that the risk of NAFLD was tightly associated with CRA. Sang et al. found that NAFLD in CRA group was significantly higher than in healthy control group, and the increased risk of NAFLD was more serious in patients with a higher prevalence of colorectal adenomatous polyps [22]. Interestingly, we found that the BMI and CAP values, and the serum levels of FPG, TG, and UA were markedly higher in the CRA patients than in healthy controls, which were consist with the previous studies. Compared to the CRA patients, the NAFLD patients and NAFLD\&CRA patients had the higher CAP values,
ALT, and AST levels, suggested that CRA acts as a risk factor for NAFLD, but could not directly lead to the development of NAFLD and NAFLD-related liver injury. No significant differences of clinical characteristics between NAFLD patient and NAFLD\&CRA patients were observed, showed that CRA did not contribute to the progression of NAFLD patients.

Since the TM6SF2 rs58542926 was found, the genetic susceptibility of this variant in NAFLD had been studied by many researchers. Increasing evidences have proven that TM6SF2 rs58542926 was an independent risk factor for NAFLD [15, 41, 42]. In our study, the TM6SF2 rs58542926 genotype distributions were significant different in NAFLD group, NAFLD\&CRA group compared to healthy control group. Besides, the genotype distributions were also different in NAFLD group and CRA group. The allele distributions of TM6SF2 rs58542926 C and T in NAFLD group and NAFLD\&CRA group were significant different compared to healthy control group. Multiple-logistic regression analysis showed that $\mathrm{CT}+\mathrm{TT}$ genotype was significant associated with the risk of NAFLD and NAFLD\&CRA, but no associated with CRA. These results suggested that TM6SF2 rs58542926 was a significant risk factor of NAFLD. CRA did not markedly affect the distributions of genotype and allele in NAFLD patients.

Hepatic TG deposition leads to the decreased serum TG level, which is the typical symptom of NAFLD patients [43]. Several studies had investigated the effects of TM6SF2 rs58542926 $\mathrm{T}$ allele on the lipid profiles. Eeva et al. conducted a study in 24,925 European populations and found that the plasma concentrations of LDL, fatty acids, TG and TC in various forms of lipoproteins in TM6SF2 rs58542926 $\mathrm{T}$ allele carriers were significant lower than in control group [44]. Elizabeth et al. confirmed that in many aspects such as crowd, animal and cell, $\mathrm{T}$ allele could reduce the fasting plasma lipids and postprandial triglycerides [45]. In our study, we investigated the effect of TM6SF2 rs58542926 T allele on the clinical characteristics in each group. In NAFLD patients, the higher CAP value and serum ALT level, and the lower serum TG level were observed in $\mathrm{T}$ allele carriers. The higher CAP value and serum AST and ALT levels, and the lower serum levels of TC, TG, and LDL-C were observed in the $\mathrm{T}$ allele carriers of NAFLD\&CRA. As the AST and ALT are the significant liver injury biomarkers [46], our results suggested that TM6SF2 rs58542926 T allele was associated with the hepatic TG accumulation, therefore leads to the decreased serum lipid profiles, and promotes the liver injury in NAFLD patients. In NAFLD\&CRA patients, TM6SF2 rs58542926 $\mathrm{T}$ allele also tightly associated with the abnormal regulation of lipids metabolism and liver injury, and the presence of CRA aggravates the clinical performance of NAFLD. 


\section{Conclusions}

In summary, we investigated the relationship of TM6SF2 rs58542926 polymorphism with the risk of NAFLD and CRA, and the effect of CRA on the TM6SF2 rs58542926 carried NAFLD patients in Han population of Qingdao district in China. Our results showed that TM6SF2 rs58542926 polymorphism was tightly associated with the risk of NAFLD and NAFLD\&CRA, but no associated with the risk of CRA. TM6SF2 rs58542926 T allele promotes the liver injury and abnormal regulation of lipids metabolism in NAFLD patients, NAFLD\&CRA patients, and overall subjects.

\section{Abbreviations \\ AASLD: American association for the study of liver disease; ALT: Alanine transaminase; AST: Aspartate aminotransferase; BMI: Body mass index; Cl: Confidence interval; CRA: Colorectal adenoma; CRC: Colorectal cancer; FBG: Fasting blood glucose; GWAS: Genome wide association studies; HCC: Hepatocellular carcinoma; HDL: High density lipoprotein cholesterol; LDL: Low density lipoprotein cholesterol; NAFL: Non-alcoholic fatty liver; NAFLD: Non-alcoholic fatty liver disease; NASH: Non-alcoholic steatohepatitis; OR: Odds ratio; PCR: Polymerase chain reaction; SNP: Single nucleotide polymorphism; TC: Total cholesterol; TG: Triglyceride; \\ TM6SF2: Transmembrane protein 6 superfamily member 2; UA: Uric acid}

\section{Acknowledgements}

We thank Qingdao Municipal Hospital, Digestive Disease Key Laboratory of Qingdao and all the participants in this study.

\section{Funding}

This study was supported by Grants of National Natural Science Foundation of China (31770837) (for the study design, data collection and analysis), the Key Research Project of Shandong Province (2016GSF201217) (for the data collection and analysis), the Qingdao, Shinan District Science and Technology Development Project Fund (2016-3-016-YY) (for the interpretation of data and manuscript writing), and the Qingdao People's Livelihood Science and technology plan (18-6-1-68-nsh) (for the manuscript writing).

\section{Availability of data and materials}

The datasets used and analyzed during the current study are available from the corresponding author on reasonable request.

\section{Authors' contributions}

LY and LSS carried out the experiment, analyzed the data, and drafted the manuscript. GYQ, MH, ZSH, and YY participated in the experiment and analyzed the data. XYN and XSY designed the research and helped to draft and write the manuscript. XYN, XSY are the guarantor. All authors reviewed the results and approved the final version of the manuscript.

\section{Ethics approval and consent to participate}

This study was approved by the Ethics Committee of Qingdao municipal hospital (approval No: 2016临审字第17号). All patients and healthy persons included in this study provided written informed consent.

\section{Consent for publication}

All patients and healthy persons included in this study provided written informed consent.

\section{Competing interests}

The authors declare that they have no competing interests.

\section{Publisher's Note}

Springer Nature remains neutral with regard to jurisdictional claims in published maps and institutional affiliations.

\section{Author details}

${ }^{1}$ Medical College of Qingdao University, Qingdao 266071, China. 2Department of Gastroenterology, Qingdao Municipal Hospital, 1 Jiaozhou Road, Qingdao 266011, Shandong Province, China. ${ }^{3}$ Department of Liver Disease, Qingdao Municipal Hospital, Qingdao 266011, China. ${ }^{4}$ Central Laboratories, Qingdao Municipal Hospital, Qingdao 266071, China. ${ }^{5}$ Digestive Disease Key Laboratory of Qingdao, Qingdao 266071, China.

Received: 24 October 2018 Accepted: 23 January 2019

Published online: 06 February 2019

\section{References}

1. Katsiki N, Mikhailidis DP, Mantzoros CS. Non-alcoholic fatty liver disease and dyslipidemia: An update. Metabolism. 2016;65(8):1109-23.

2. Clark JM. The epidemiology of nonalcoholic fatty liver disease in adults. J Clin Gastroenterol. 2006;40(Suppl 1):S5-10

3. Weiss J, Rau M, Geier A. Non-alcoholic fatty liver disease: epidemiology, clinical course, investigation, and treatment. Dtsch Arztebl Int. 2014;111(26): 447-52.

4. Lu ZY, Shao Z, Li YL, Wulasihan M, Chen XH. Prevalence of and risk factors for non-alcoholic fatty liver disease in a Chinese population: An 8-year follow-up study. World J Gastroenterol. 2016:22(13):3663-9.

5. Wang X, Liu Z, Wang K, Wang Z, Sun X, Zhong L, Deng G, Song G, Sun B, Peng $Z$, et al. Additive effects of the risk alleles of PNPLA3 and TM6SF2 on non-alcoholic fatty liver disease (NAFLD) in a Chinese population. Front Genet. 2016;7:140.

6. Xu R, Tao A, Zhang S, Deng Y, Chen G. Association between patatin-like phospholipase domain containing 3 gene (PNPLA3) polymorphisms and nonalcoholic fatty liver disease: a HuGE review and meta-analysis. Sci Rep. 2015:5:9284.

7. Bechmann LP, Gastaldelli A, Vetter D, Patman GL, Pascoe L, Hannivoort RA, Lee UE, Fiel I, Munoz U, Ciociaro D, et al. Glucokinase links Kruppel-like factor 6 to the regulation of hepatic insulin sensitivity in nonalcoholic fatty liver disease. Hepatology. 2012;55(4):1083-93.

8. Tan HL, Zain SM, Mohamed R, Rampal S, Chin KF, Basu RC, Cheah PL, Mahadeva S, Mohamed Z. Association of glucokinase regulatory gene polymorphisms with risk and severity of non-alcoholic fatty liver disease: an interaction study with adiponutrin gene. J Gastroenterol. 2014;49(6):1056-64.

9. Yuan C, Lu L, An B, Jin W, Dong Q, Xin Y, Xuan S. Association between LYPLAL1 rs12137855 polymorphism with ultrasound-defined nonalcoholic fatty liver disease in a Chinese Han population. Hepat Mon. 2015;15(12):e33155

10. Leon-Mimila P, Vega-Badillo J, Gutierrez-Vidal R, Villamil-Ramirez H, VillarealMolina T, Larrieta-Carrasco E, Lopez-Contreras BE, Kauffer LR, MaldonadoPintado DG, Mendez-Sanchez N, et al. A genetic risk score is associated with hepatic triglyceride content and non-alcoholic steatohepatitis in Mexicans with morbid obesity. Exp Mol Pathol. 2015;98(2):178-83.

11. Kozlitina J, Smagris E, Stender S, Nordestgaard BG, Zhou HH, TybjaergHansen A, Vogt TF, Hobbs HH, Cohen JC. Exome-wide association study identifies a TM6SF2 variant that confers susceptibility to nonalcoholic fatty liver disease. Nat Genet. 2014;46(4):352-6.

12. Chen LZ, Xia HH, Xin YN, Lin ZH, Xuan SY. TM6SF2 E167K variant, a novel genetic susceptibility variant, contributing to nonalcoholic fatty liver disease. J Clin Transl Hepatol. 2015:3(4):265-70.

13. Musso G, Cipolla U, Cassader M, Pinach S, Saba F, De Michieli F, Paschetta E, Bongiovanni D, Framarin L, Leone N, et al. TM6SF2 rs58542926 variant affects postprandial lipoprotein metabolism and glucose homeostasis in NAFLD. J Lipid Res. 2017;58(6):1221-9.

14. Grandone A, Cozzolino D, Marzuillo P, Cirillo G, Di Sessa A, Ruggiero L, Di Palma MR, Perrone L, Miraglia Del Giudice E. TM6SF2 Glu167Lys polymorphism is associated with low levels of LDL-cholesterol and increased liver injury in obese children. Pediatr Obes. 2016;11(2):115-9.

15. Wang X, Liu Z, Peng Z, Liu W. The TM6SF2 rs58542926 T allele is significantly associated with non-alcoholic fatty liver disease in Chinese. J Hepatol. 2015;62(6):1438-9.

16. Wong W, Wong GL, Tse CH, Chan HL. Prevalence of the TM6SF2 variant and non-alcoholic fatty liver disease in Chinese. J Hepatol. 2014;61(3):708-9.

17. Arnold M, Sierra MS, Laversanne M, Soerjomataram I, Jemal A, Bray F. Global patterns and trends in colorectal cancer incidence and mortality. Gut. 2017; 66(4):683-91. 
18. Sawbridge D, Probert C. Population-based screening in colorectal cancer current practice and future developments: faecal biomarkers review. J Gastrointestin Liver Dis. 2014;23(2):195-202.

19. Zauber AG, Winawer SJ, O'Brien MJ, Lansdorp-Vogelaar I, van Ballegooijen M, Hankey BF, Shi W, Bond JH, Schapiro M, Panish JF, et al. Colonoscopic polypectomy and long-term prevention of colorectal-cancer deaths. N Engl J Med. 2012;366(8):687-96.

20. Sweetser S, Smyrk TC, Sinicrope FA. Serrated colon polyps as precursors to colorectal cancer. Clin Gastroenterol Hepatol. 2013;11(7):760-7 quiz e754-765.

21. Ding W, Fan J, Qin J. Association between nonalcoholic fatty liver disease and colorectal adenoma: a systematic review and meta-analysis. Int J Clin Exp Med. 2015;8(1):322-33.

22. Hwang ST, Cho YK, Park JH, Kim HJ, Park DI, Sohn Cl, Jeon WK, Kim BI, Won $\mathrm{KH}$, Jin W. Relationship of non-alcoholic fatty liver disease to colorectal adenomatous polyps. J Gastroenterol Hepatol. 2010;25(3):562-7.

23. Lee YI, Lim YS, Park HS. Colorectal neoplasms in relation to non-alcoholic fatty liver disease in Korean women: a retrospective cohort study. J Gastroenterol Hepatol. 2012;27(1):91-5.

24. Chen QF, Zhou XD, Sun YJ, Fang DH, Zhao Q, Huang JH, Jin Y, Wu JS. Sex-influenced association of non-alcoholic fatty liver disease with colorectal adenomatous and hyperplastic polyps. World J Gastroenterol. 2017;23(28):5206-15

25. Galbraith RL, Poole EM, Duggan D, Muehling J, Hsu L, Makar K, Xiao L, Potter JD, Ulrich CM. Polymorphisms in WNT6 and WNT10A and colorectal adenoma risk. Nutr Cancer. 2011;63(4):558-64.

26. Yin G, Hamajima N, Morita M, Tajima O, Tabata S, Kono S. Lack of influence of the ADH1B Arg47His genetic polymorphism on risk of colorectal adenoma in middle-aged Japanese men. Asian Pac J Cancer Prev. 2011; 12(1):297-302.

27. Gilsing AM, Berndt SI, Ruder EH, Graubard BI, Ferrucci LM, Burdett L, Weissfeld JL, Cross AJ, Sinha R. Meat-related mutagen exposure, xenobiotic metabolizing gene polymorphisms and the risk of advanced colorectal adenoma and cancer. Carcinogenesis. 2012;33(7):1332-9.

28. Rickham PP. Human Experimentation. Code of ethics of the world medical association. Declaration Of Helsinki. Br Med J. 1964;2(5402):177.

29. Fan JG, Jia JD, Li YM, Wang BY, Lu LG, Shi JP, Chan LY. Guidelines for the diagnosis and management of nonalcoholic fatty liver disease: update 2010: (published in Chinese on Chinese journal of hepatology 2010; 18:163-166). J Dig Dis. 2011;12(1):38-44.

30. An BQ, Lu LL, Yuan C, Xin YN, Xuan SY. Leptin receptor gene polymorphisms and the risk of non-alcoholic fatty liver disease and coronary atherosclerosis in the Chinese Han population. Hepat Mon. 2016; 16(4):e35055.

31. Mota M, Banini BA, Cazanave SC, Sanyal AJ. Molecular mechanisms of lipotoxicity and glucotoxicity in nonalcoholic fatty liver disease. Metabolism. 2016;65(8):1049-61.

32. Anstee QM, Day CP. The genetics of NAFLD. Nat Rev Gastroenterol Hepatol. 2013;10(11):645-55.

33. Rinella ME, Sanyal AJ. NAFLD in 2014: genetics, diagnostics and therapeutic advances in NAFLD. Nat Rev Gastroenterol Hepatol. 2015;12(2):65-6.

34. Rowell RJ, Anstee QM. An overview of the genetics, mechanisms and management of NAFLD and ALD. Clin Med (Lond). 2015;15(Suppl 6):s77-82.

35. Anstee QM, Day CP. The genetics of nonalcoholic fatty liver disease: spotlight on PNPLA3 and TM6SF2. Semin Liver Dis. 2015;35(3):270-90.

36. Sanna C, Rosso C, Marietti M, Bugianesi E. Non-alcoholic fatty liver disease and extra-hepatic cancers. Int J Mol Sci. 2016;17(5).

37. Moon CM, Yun KE, Ryu S, Chang Y, Park DI. High serum alanine aminotransferase is associated with the risk of colorectal adenoma in Korean men. J Gastroenterol Hepatol. 2017;32(7):1310-7.

38. Dietrich P, Hellerbrand C. Non-alcoholic fatty liver disease, obesity and the metabolic syndrome. Best Pract Res Clin Gastroenterol. 2014;28(4):637-53.

39. Mikolasevic I, Orlic L, Franjic N, Hauser G, Stimac D, Milic S. Transient elastography (FibroScan ((R))) with controlled attenuation parameter in the assessment of liver steatosis and fibrosis in patients with nonalcoholic fatty liver disease - where do we stand? World J Gastroenterol. 2016;22(32):7236-51.

40. Castera L, Vilgrain V, Angulo P. Noninvasive evaluation of NAFLD. Nat Rev Gastroenterol Hepatol. 2013;10(11):666-75.

41. Zhou Y, Llaurado G, Oresic M, Hyotylainen T, Orho-Melander M, Yki-Jarvinen $\mathrm{H}$. Circulating triacylglycerol signatures and insulin sensitivity in NAFLD associated with the E167K variant in TM6SF2. J Hepatol. 2015;62(3):657-63.
42. Sookoian S, Castano GO, Scian R, Mallardi P, Fernandez Gianotti T, Burgueno AL, San Martino J, Pirola CJ. Genetic variation in transmembrane 6 superfamily member 2 and the risk of nonalcoholic fatty liver disease and histological disease severity. Hepatology. 2015;61(2):515-25.

43. Lee J, Kim Y, Friso S, Choi SW. Epigenetics in non-alcoholic fatty liver disease. Mol Asp Med. 2017:54:78-88.

44. Sliz E, Sebert S, Wurtz P, Kangas AJ, Soininen P, Lehtimaki T, Kahonen M, Viikari J, Mannikko M, Ala-Korpela M, et al. NAFLD risk alleles in PNPLA3, TM6SF2, GCKR and LYPLAL1 show divergent metabolic effects. Hum Mol Genet. 2018;27(12):2214-23.

45. O'Hare EA, Yang R, Yerges-Armstrong LM, Sreenivasan U, McFarland R, Leitch CC, Wilson MH, Narina S, Gorden A, Ryan KA, et al. TM6SF2 rs58542926 impacts lipid processing in liver and small intestine. Hepatology. 2017;65(5):1526-42.

46. McGill MR. The past and present of serum aminotransferases and the future of liver injury biomarkers. EXCLI J. 2016;15:817-28.
Ready to submit your research? Choose BMC and benefit from:

- fast, convenient online submission

- thorough peer review by experienced researchers in your field

- rapid publication on acceptance

- support for research data, including large and complex data types

- gold Open Access which fosters wider collaboration and increased citations

- maximum visibility for your research: over 100M website views per year

At BMC, research is always in progress.

Learn more biomedcentral.com/submissions 